\title{
Two-body loss rates for reactive collisions of cold atoms
}

\author{
C. Cop ${ }^{*}$ and R. Walser \\ TU Darmstadt, Institut für Angewandte Physik, D-64289 Darmstadt, Germany
}

(Dated: January 10, 2018)

\begin{abstract}
We present an effective two-channel model for reactive collisions of cold atoms. It augments elastic molecular channels with an irreversible, inelastic loss channel. Scattering is studied with the distorted-wave Born approximation and yields general expressions for angular momentum resolved cross sections as well as two-body loss rates. Explicit expressions are obtained for piece-wise constant potentials. A pole expansion reveals simple universal shape functions for cross sections and twobody loss rates in agreement with the Wigner threshold laws. This is applied to collisions of metastable ${ }^{20} \mathrm{Ne}-{ }^{21} \mathrm{Ne}$ atoms, which decay primarily through exothermic Penning-, or Associativeionization processes. From a numerical solution of the multichannel Schrödinger equation using the best currently available molecular potentials, we have obtained synthetic scattering data. Using the two-body loss shape functions derived in this paper, we can match these scattering data very well.
\end{abstract}

\section{INTRODUCTION}

Understanding reactive collisions of metastable rare gas atoms $\left(\mathrm{Rg}^{*}\right)$ is a central topic in trapping such species. Major loss processes in $\mathrm{Rg}^{*}$ collisions are Penning Ionization (PI) and Associative Ionization (AI) [1, 2]

$$
\begin{array}{ll}
\mathrm{PI}: & \mathrm{Rg}^{*}+\mathrm{Rg}^{*} \longrightarrow \mathrm{Rg}+\mathrm{Rg}^{+}+e^{-}, \\
\mathrm{AI}: & \mathrm{Rg}^{*}+\mathrm{Rg}^{*} \longrightarrow \mathrm{Rg}_{2}^{+}+e^{-} .
\end{array}
$$

In current trapping experiments [3, it is possible to observe the reaction kinetic with high resolution and in real-time, as the ionic fragments can be detected with single-ion precision.

In order to parametrize cold ionizing collisions such as PI and AI, different models have been presented. The quantum reflection model 4] has been applied successfully to explain two-body loss rates in cold collisions of metastable rare gas atoms, for $\mathrm{He}^{*}$ collisions [5] 7], $\mathrm{Xe}^{*}$ collisions [8] and $\mathrm{Kr}^{*}$ collisions [9]. This model assumes complete ionization at short-range and predicts universal scattering rates for collisions of different isotope mixtures in agreement with mass scaling.

Current cold Ne* experiments of G. Birkl et al. [10 12. have provided new data on elastic as well as inelastic scattering. The isotope composition of the Ne gas consists of the bosonic (B ) and fermionic (F) isotopes with natural abundance, i.e. ${ }^{20} \mathrm{Ne}(90.48 \%, \mathrm{~B}),{ }^{21} \mathrm{Ne}(0.27 \%$, F), ${ }^{22} \mathrm{Ne}(9.25 \%, \mathrm{~B})$ [13. Homonuclear [10, 12] and heteronuclear collision rates [11] were obtained, preparing polarized as well as unpolarized subensembles with respect to the internal magnetic substates. The experiment uncovers deviations from a fundamental mass scaling law for the elastic and inelastic scattering rates of different isotopes. Thus, a simple quantum reflection model is insufficient.

* christiancop@online.de

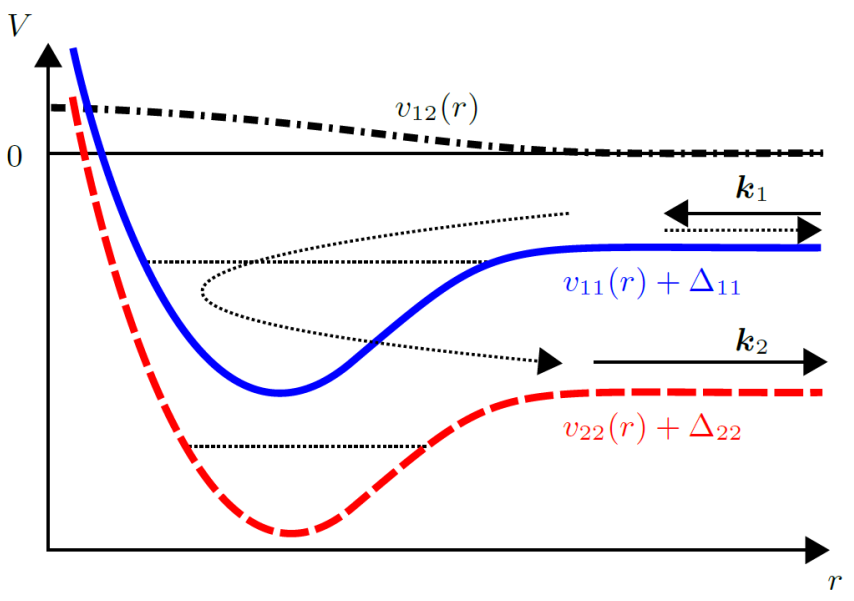

Figure 1. (Color online) Effective two-channel reaction model: molecular scattering channel 1 coupled to loss channel 2. Radial molecular potentials $v_{11}(r)$ (solid, blue) and loss potential $v_{22}(r)$ (dashed, red) are coupled by $v_{12}(r)$ (dash-dotted, black). At large separations, the potentials approach the channel thresholds $\Delta_{i i}$ with $\Delta_{11}>\Delta_{22}$ and all couplings $v_{i j}(r)$ vanish. The straight thin dashed black lines indicate bound states.

To match these experimental facts, we consider an effective two-channel model using molecular potentials [14, 15] with van-der-Waals interaction at long-range [16] for the elastic channels as shown in Fig. 1. The free parameters of the fictitious inelastic loss potential, as well as the coupling strength were obtained from a finite temperature fit to the scattering data with very good agreement. The threshold scattering rates of the numerical two-channel model are also in very good agreement with recent non-universal models for reactive collisions [17. 19, which have been applied successfully to atom-ion collisions [20, polar molecule collisions [21 25] and collisions of other species [26]. For details, we refer to Ref. [27].

In this article, we will complement the numerical approach with an analytical study of the two-channel model using piecewise constant couplings and potentials, only. Within this hypothesis, scattering can be studied ana- 
lytically in the complex $k$ - or $E$-plane. The coupling strength between the molecular channel and the loss channel should be weak on physical grounds. Therefore, we will employ the distorted-wave Born approximation (DWBA), which is introduced in Sec. II and yields a general expression for the partial-wave resolved two-body loss rates. Moreover, we introduce the concepts of the pole expansion of the $S$-matrix. Coupled square-wells are an illuminating application of the previous discussion. Exact and DBWA solutions are presented in Sec. III] However, even this simple model yields explicit expressions, which are too complex for interpretation. Therefore, we will step back to single channel scattering in Sec. [V] and revisit the well known results $s-, p$-, and $d$-wave scattering phases and cross sections [28]. Already there, the complex analytical expression conceal the essential physics, which is uncovered only by a pole expansion. In Sec. $\mathrm{V}$, we merge the insights from the previous sections and study the two-body loss rates. Exact square-well results for $s^{-}, p$-, and $d$-waves are compared to explicit expressions (shape functions) using the pole approximation. These shape functions are consistent with the Wigner threshold laws and are depicted for some suitably chosen parameters. Finally, in Sec. VI. we demonstrate the utility of these physically motivated shape functions. While originally derived for square well potentials, they are also very well suited to interpolate synthetic scattering data obtained from the two-channel van-der-Waals model for the ${ }^{20} \mathrm{Ne}^{21} \mathrm{Ne}$ collisions [27]. The article ends with conclusions and two appendices regarding definitions of Riccati-Bessel functions and details on the pole expansion in the complex plane.

\section{MULTICHANNEL SCATTERING}

In order to understand the reactive quantum kinetics of Eqs. (1) quantitatively, one needs to consider two coupled channels, as depicted in Fig. 1. There, a molecular state manifold or channel 1 can either scatter elastically within the manifold, or inelastically to a loss channel 2 . This removes particles irreversibly from the interaction zone. In the rest-frame of the collision partners, the state manifold $\left.\mathscr{H}=\operatorname{span}\left\{\boldsymbol{k} \in \mathbb{R}^{3}, i \in\{1,2\} \| \boldsymbol{k}, i\right\rangle\right\}$ is spanned by plane-waves decorated with a channel subscript. The energy

$$
H=H_{0}+V(r)
$$

consists of a asymptotically free Hamiltonian operator $H_{0}$ and a short range, matrix-valued potential $V(r)$. To keep the notation compact, we will use natural units $\hbar=$ $2 \mu=1$, with the reduced mass of the collision partners $\mu=m_{1} m_{2} /\left(m_{1}+m_{2}\right)$. Thus, $H_{0}=\boldsymbol{p}^{2}+\Delta$ denotes the relative kinetic energy of the collision partners with respect to the collision thresholds energies $\Delta_{i j}=\delta_{i j} \Delta_{i i}$ with $\Delta_{11}>\Delta_{22}$. Plane-waves are eigenfunctions of the non-interacting two-particle system

$$
H_{0}\left|\boldsymbol{k}_{i}, i\right\rangle=E\left|\boldsymbol{k}_{i}, i\right\rangle, \quad E=k_{i}^{2}+\Delta_{i i},
$$

with channel wave-numbers $k_{i}(E)=\sqrt{E-\Delta_{i i}}$ determined by the energy $E$ relative to the threshold energies $\Delta_{i i}$. The short range molecular potential

$$
V=V^{\mathrm{I}}+V^{\mathrm{II}}=\left(\begin{array}{cc}
v_{11} & 0 \\
0 & v_{22}
\end{array}\right)+\left(\begin{array}{cc}
0 & v_{12} \\
v_{12}^{*} & 0
\end{array}\right)
$$

can be decomposed into the potentials $V^{\mathrm{I}}$ for each individual channel and their coupling $V^{\mathrm{II}}$.

\section{A. Two-potential formula for the T-matrix}

Scattering in the presence of two potentials can be described using the two-potential formula 29] for the $T$ matrix elements

$$
\begin{gathered}
t_{i j}\left(\boldsymbol{k}_{i}, \boldsymbol{k}_{j}\right)=\left\langle\boldsymbol{k}_{i}, i|T| \boldsymbol{k}_{j}, j\right\rangle \\
=\left\langle\boldsymbol{k}_{i}, i\left|V^{\mathrm{I}}\right| \boldsymbol{k}_{j}, j,+{ }^{\mathrm{I}}\right\rangle+\left\langle\boldsymbol{k}_{i}, i,-{ }^{\mathrm{I}}\left|V^{\mathrm{II}}\right| \boldsymbol{k}_{j}, j,+\right\rangle .
\end{gathered}
$$

This expression introduces the scattering states $\left|\boldsymbol{k}_{i}, i, \pm^{\mathrm{I}}\right\rangle$ that are obtained in the absence of potential $V^{\mathrm{II}}$ and the fully coupled scattering states $\left|\boldsymbol{k}_{i}, i, \pm\right\rangle$. They are defined by their corresponding Lippmann-Schwinger equations as

$$
\begin{aligned}
\left|\boldsymbol{k}_{i}, i, \pm^{\mathrm{I}}\right\rangle & =\left|\boldsymbol{k}_{i}, i\right\rangle+G_{0}^{ \pm}(E)_{i i} v_{i i}^{\mathrm{I}}\left|\boldsymbol{k}_{i}, i, \pm^{\mathrm{I}}\right\rangle \\
\left|\boldsymbol{k}_{i}, i, \pm\right\rangle & =\left|\boldsymbol{k}_{i}, i\right\rangle+\sum_{j=1}^{2} G_{0}^{ \pm}(E)_{i i} v_{i j}\left|\boldsymbol{k}_{j}, j, \pm\right\rangle,
\end{aligned}
$$

with $G_{0}^{ \pm}(E) \equiv \lim _{\epsilon \rightarrow+0}\left(E \pm i \epsilon-H_{0}\right)^{-1}$ denoting the free advanced (-) and retarded (+) Green's functions.

The exact result Eq. (5) is most useful if the second potential is weak and the Born series expansion is applicable. To first order, the distorted-wave Born approximation for the T-matrix reads

$$
\begin{aligned}
& t_{i j}\left(\boldsymbol{k}_{i}, \boldsymbol{k}_{j}\right)=t_{i j}^{(0)}\left(\boldsymbol{k}_{i}, \boldsymbol{k}_{j}\right)+t_{i j}^{(1)}\left(\boldsymbol{k}_{i}, \boldsymbol{k}_{j}\right)+\odot\left(V^{\mathrm{II}^{2}}\right) \\
& =\left\langle\boldsymbol{k}_{i}, i\left|V^{\mathrm{I}}\right| \boldsymbol{k}_{j}, j,+{ }^{\mathrm{I}}\right\rangle+\left\langle\boldsymbol{k}_{i}, i,-{ }^{\mathrm{I}}\left|V^{\mathrm{I}}\right| \boldsymbol{k}_{j}, j,+{ }^{\mathrm{I}}\right\rangle+\ldots
\end{aligned}
$$

It is important to note that first order corrections due to the second potential only occur in the off-diagonal $t_{i \neq j}$ matrix element.

\section{B. Scattering states in terms of Jost functions}

To proceed in the analysis of reactive two-body scattering, we will assume rotational invariant interactions [27]. This implies angular momentum conservation and suggests to use symmetry adapted, spherical coordinates $(r, \theta, \phi)$. Single channel scattering states with outgoing- 
or incoming asymptotics $( \pm)$ are conventionally [29] defined as

$$
\langle\mathbf{x} \mid E, l, m, \pm\rangle=i^{l}\left(\frac{2}{\pi \sqrt{E}}\right)^{1 / 2} \frac{\psi_{l}^{ \pm}(E, r)}{r} Y_{l m}(\theta, \phi)
$$

with the reduced scalar wave-functions $\psi_{l}^{ \pm}(E, r)$, the eigenvalues of the angular projection $m$ on the quantization axis $z$ and angular momentum $l$. If this is generalized to multichannel scattering, the Schrödinger Eq. (2) reads

$$
\left(K^{2}(E)+\partial_{r}^{2}-V(r)-\frac{l(l+1)}{r^{2}}\right) \Psi_{l}^{ \pm}(E, r)=0
$$

where the wave-number matrix $K_{i j}(E)=\delta_{i j} k_{i}(E)$ is defined by the individual channel wave-numbers $k_{i}(E)$ as in Eq. (3). In turn, the reduced scattering wave-function

$$
\Psi_{l}^{ \pm}(E, r)=\left(\begin{array}{ll}
\psi_{l, 11}^{ \pm}(E, r) & \psi_{l, 12}^{ \pm}(E, r) \\
\psi_{l, 21}^{ \pm}(E, r) & \psi_{l, 22}^{ \pm}(E, r)
\end{array}\right)
$$

becomes a $2 \times 2$ matrix to account for the two linearly independent solutions in the scattering channels. They are determined from the boundary condition at the origin

$$
\Psi_{l}^{ \pm}(E, r=0)=0
$$

and the asymptotic behavior for the outgoing solution

$$
\Psi_{l}^{+}(E, r) \underset{r \rightarrow \infty}{\longrightarrow} \frac{i}{2}\left\{\hat{h}_{l}^{-}(K r)-\frac{\hat{h}_{l}^{+}(K r)}{\sqrt{K}} S_{l}(E) \sqrt{K}\right\},
$$

as well as $\Psi_{l}^{-}=\left(\Psi_{l}^{+}\right)^{\dagger}$. Here, $\hat{h}_{l}^{ \pm}$are the Riccati-Hankel functions as defined in appendix $\mathrm{A}$ and $S_{l}(E)$ denotes the $S$-matrix with angular momentum $l$.

The regular solution $\Phi_{l}(E, r)$ is another solution to the Schrödinger equation Eq. 110. It is defined by the boundary condition at the origin

$$
\Phi_{l}(E, r) \underset{r \rightarrow 0}{\longrightarrow} \hat{\jmath}_{l}(K r) .
$$

In each channel, the solution approaches a Riccati-Bessel function $\hat{\jmath}_{l}\left(k_{i} r\right)$ close to the origin and is smaller in amplitude in all other coupled channels (cf. appendix A). Since the coupled radial Schrödinger equation Eq. 10 and the boundary condition Eq. 14 are real for real energies, the regular solution is real as well. In the asymptotic region the regular solution reads

$$
\Phi_{l}(E, r) \underset{r \rightarrow \infty}{\longrightarrow} \frac{i}{2}\left\{\hat{h}_{l}^{-}(K r) \mathscr{F}_{l}(K)-\hat{h}_{l}^{+}(K r) \mathscr{F}_{l}(-K)\right\},
$$

with the Jost matrix $\mathscr{F}_{l}(K)$ and its matrix elements $f_{l, i j}$. Comparing the asymptotic form of the scattering wavefunction Eq. (13) to the asymptotic form of the regular solution Eq. (15) leads to the relation of the scattering wave-function and the regular solution, given by

$$
\psi_{l}^{+}(E, r)=\Phi_{l}(E, r) \mathscr{F}_{l}^{-1}(K),
$$

and to the relation of the Jost matrix and the $S$-matrix

$$
S_{l}(E)=\sqrt{K} \mathscr{F}_{l}(-K) \mathscr{F}_{l}^{-1} / \sqrt{K} .
$$

Using the constitutive relation between the $S$ and the $T$ operator

$$
S(E)=\mathbb{1}-2 \pi i \delta\left(E-H_{0}\right) T(E),
$$

one can connect all relevant entities. Within the distorted-wave Born approximation from Eq. (8), the $S$ matrix reads to first order as

$$
S_{l}(E)=\left(\begin{array}{cc}
s_{l, 11}^{(0)}(E) & s_{l, 12}^{(1)}(E) \\
s_{l, 21}^{(1)}(E) & s_{l, 22}^{(0)}(E)
\end{array}\right)+\odot\left(V^{\mathrm{II}^{2}}\right),
$$

where the diagonal $S$-matrix elements

$$
s_{l, i i}^{(0)}(E)=\frac{f_{l, i i}^{(0)}\left(-k_{i}(E)\right)}{f_{l, i i}^{(0)}\left(k_{i}(E)\right)}=e^{2 i \eta_{l, i}\left(k_{i}(E)\right)} .
$$

are the uncoupled solutions for the potential $V_{\mathrm{I}}$ and given in terms of the uncoupled Jost functions $f_{l, i i}^{(0)}\left(k_{i}\right)$, or equivalently by scattering phases $\eta_{l, i}\left(k_{i}\right)$. For the sake of readability, we have not included a further superscript (0) in the scattering phase. The scattering phases

$$
\eta_{l, i}\left(k_{i}(E)\right)=\eta_{l, i}^{b}\left(k_{i}(E)\right)+\eta_{l, i}^{r}\left(k_{i}(E)\right)
$$

can be decomposed into a background contribution $\eta_{l, i}^{b}$, which is a slowly varying function of $k_{i}$ and a resonant contribution $\eta_{l, i}^{r}$, which changes rapidly across a resonance. With this decomposition we can write for the $S$-matrix elements

$$
s_{l, i i}^{(0)}(E)=s_{l, i i}^{(0) b}(E) \cdot s_{l, i i}^{(0) r}(E) .
$$

In the following, we prefer to switch from the energy parameter $E$ to the wave-number $k$. All functions will become functions of $k_{i}(E)$ while being implicitly functions of $E$ due to Eq. (3). The observable available for measurement is the partial elastic scattering cross section 29]

$$
\sigma_{l, i i}^{(0)}\left(k_{i}\right)=2 \pi \frac{2 l+1}{k_{i}^{2}}\left(1-\operatorname{Re} s_{l, i i}^{(0)}\left(k_{i}\right)\right) .
$$

The cross section for scattering of channel 1 to channel 2 in the DWBA is given by

$$
\sigma_{l, 12}^{(1)}\left(k_{1}\right)=\pi \frac{2 l+1}{k_{1}^{2}}\left|s_{l, 12}^{(1)}\left(k_{1}\right)\right|^{2} .
$$

The off-diagonal $S$ matrix-elements are obtained from the DWBA approximation Eq. (8) and the constitutive relation Eq. (18) as

$$
\begin{aligned}
s_{l, 12}^{(1)}(E) & =\frac{2 \int_{0}^{\infty} d r \psi_{l, 11}^{(0)+}(E, r) v_{12}^{\mathrm{II}}(r) \psi_{l, 22}^{(0)+}(E, r)}{i \sqrt{k_{1} k_{2}}} \\
& =e^{2 i \eta_{l, 12}\left(k_{1}(E)\right)}
\end{aligned}
$$


where $\psi_{l, i i}^{(0)+}(E, r)$ are the uncoupled solutions of the radial Schrödinger Eq. 10 for the potential $V^{\mathrm{I}}$ only, using $\left(\psi_{l, i i}^{(0)-}(E)\right)^{*}=\psi_{l, i i}^{(0)+}(E)$. The complex phase $\eta_{l, 12}\left(k_{1}\right) \in$ $\mathbb{C}$ considers also attentuation and can be decomposed into a resonance- and a background contribution as in Eq. (21).

The observable for measurement of atom loss in channel 1 is the two-body loss rate [8] which is given in terms of the cross section from channel 1 to channel 2

$$
\beta_{l}^{(1)}\left(k_{1}\right)=2 k_{1} \sigma_{l, 12}^{(1)}\left(k_{1}\right)=2 \pi \frac{2 l+1}{k_{1}}\left|s_{l, 12}^{(1)}\left(k_{1}\right)\right|^{2} .
$$

It quantifies the loss of probability current from the elastic scattering channel 1.

\section{Pole expansion of the $S$-matrix}

Scattering theory greatly benefits from complex analysis and the continuation of real parameters, like the energy $E$ or the wave-number $k$, into the complex plane. For finite-range potentials, it can be shown that the Jost functions are entire functions of $k$. Then, the $S$-matrix is analytic everywhere in the complex $E$-, or $k$-plane except at singular points, when the Jost function vanishes [29].

For complex channel wave-numbers $k_{i}$, the analytic continuation of the uncoupled Jost functions reads

$$
f_{l}(k)^{*}=f_{l}\left(-k^{*}\right) .
$$

With the help of the Weierstrass factorization theorem [30 32 , one can express the Jost function

$$
\begin{aligned}
f_{l}(k)= & f_{l}(0) e^{i k} \prod_{n=1}^{N^{\prime}}\left(1-i \frac{k}{\varkappa_{n}}\right) \prod_{n=1}^{N}\left(1+i \frac{k}{\mathscr{K}_{n}}\right) \\
& \times \prod_{n=1}^{\infty}\left(1+\frac{k}{k_{n}^{*}}\right)\left(1-\frac{k}{k_{n}}\right),
\end{aligned}
$$

as an infinite product of its zeros. For each of the $N^{\prime}$ virtual states of the scattering potential, there is a zero of the Jost function on the negative imaginary axis at $k=-i \varkappa_{n}$ with $\varkappa_{n}>0$. For each of the $N$ bound states, the Jost function vanishes on the positive imaginary axis at $k=i \mathscr{K}_{n}$ with $\mathscr{K}_{n}>0$. Moreover, there are always infinitely many scattering resonances of the potential, which correspond to the zeros of the Jost function at $k=k_{n}$ and $k=-k_{n}^{*}$ with $\operatorname{Re} k_{n}>0$.

From this representation of the Jost function, one obtains the $S$-matrix of Eq. (20) as an infinite product of poles in the complex $k=k(E)$ plane

$$
\begin{aligned}
s_{l}(E)= & e^{-2 i k} \prod_{n=1}^{N^{\prime}} \frac{\varkappa_{n}+i k}{\varkappa_{n}-i k} \prod_{n=1}^{N} \frac{\mathscr{K}_{n}-i k}{\mathscr{K}_{n}+i k} \prod_{n=1}^{\infty} \frac{k_{n}+k}{k_{n}^{*}+k} \frac{k_{n}^{*}-k}{k_{n}-k} \\
= & e^{2 i \eta_{l}^{b}(k)} s_{l}^{r}(E) .
\end{aligned}
$$

For low-energy collisions, the analytical behavior of the $S$-matrix is dominated by the resonant contribution $s_{l}^{r}$ originating from states closes to the energy threshold. The effect of the infinitely many other poles of the pole expansion can be summarized in a slowly varying background scattering phase $\eta_{l}^{b}$.

\section{COUPLED SQUARE-WELLS}

In order to put these general considerations to practical use, we will study an elementary example of a weakly coupled square-well potential, which is shown in Fig. 2. There, the piecewise constant potential matrix reads

$$
v_{i j}(r)= \begin{cases}v_{i j} & r \leq 1 \\ \Delta_{i j} & r>1\end{cases}
$$

The two levels $i \in\{1,2\}$ are coupled within a radius $r<1$ and decouple outside $\Delta_{i \neq j}=0$. The depths of the attractive potentials are parametrized by $\kappa_{i}$ and the inner level spacing is conveniently abbreviated by $\delta v$ as

$$
\begin{aligned}
& \kappa_{i}^{2}=\Delta_{i i}-v_{i i}>0 \\
& \delta v=v_{11}-v_{22}
\end{aligned}
$$

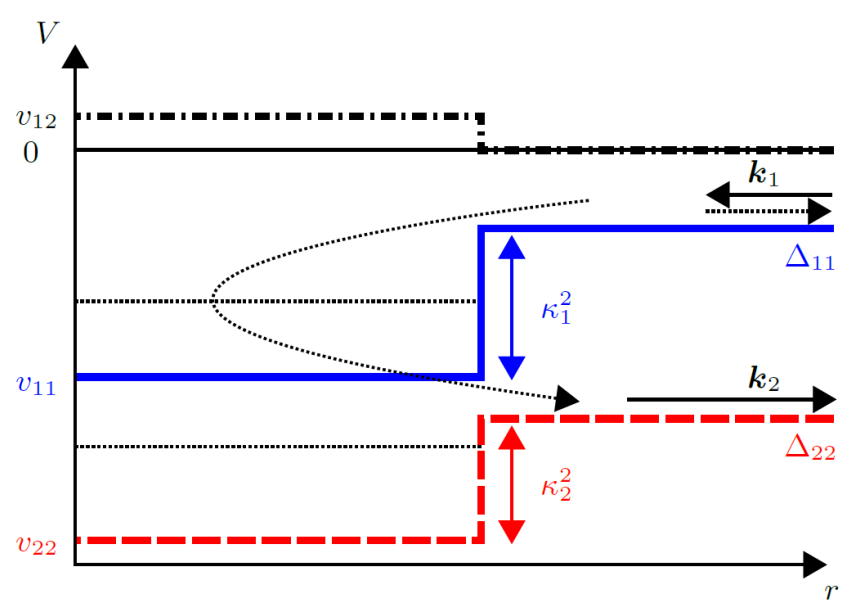

Figure 2. (Color online) Effective two-channel square-well potential vs. radius $r$. Within the range $r<1$, the potentials $v_{11}$ (solid, blue), $v_{22}$ (dashed, red) and $v_{12}$ (dash-dotted, black) are constant. For radii $r>1$, the potential matrix decouples into the channel thresholds $\Delta_{11}$ and $\Delta_{22}$. The channel wavenumbers $k_{1}, k_{2}$ are defined as in Eq. (3). The straight thin dashed black lines indicate bound states.

The utility of the model is seen by solving it perturbatively in Sec. III A and exactly in Sec. IIIB. The complexity of the solution conceals resonances and a simple interpretation is possible only by the pole expansion presented in Sec. IV eventually. 


\section{A. Distorted-wave Born approximation}

First, to initialize the DWBA, we need the solution for the uncoupled square-well Schrödinger Eq. 10 with $v_{12}=0$. In the inner and outer region the solution reads

$$
\psi_{l, i i}^{(0)+}(E, r)= \begin{cases}\left(\frac{k_{i}}{q_{i}}\right)^{l+1} \frac{\hat{\jmath}_{l}\left(q_{i} r\right)}{\hat{f}_{l, i i}^{(0)}\left(k_{i}\right)}, & r \leq 1, \\ \frac{s_{l, i i}^{(0)}(E) \hat{h}_{l}^{+}\left(k_{i} r\right)-\hat{h}_{l}^{-}\left(k_{i} r\right)}{2 i}, & r>1,\end{cases}
$$

where we have introduced channel wave-numbers $q_{i}$ and $k_{i}$ for the inner and outer regions

$$
\begin{aligned}
& q_{1}^{2} \equiv E-v_{11}=k_{1}^{2}+\kappa_{1}^{2}, \\
& q_{2}^{2} \equiv E-v_{22}=k_{2}^{2}+\kappa_{2}^{2},
\end{aligned}
$$

and $k_{i}$ as in Eq. (3). The ansatz for the wave-function of Eq. 333 introduces the Jost function $f_{l, i i}^{(0)}\left(k_{i}\right)$ by the relation between the regular solution at the origin Eq. (14) and the scattering solution Eq. (16). By matching the wave-function smoothly at $r=1$, one obtains the Jost function $f_{l, i i}^{(0)}\left(k_{i}\right)=g_{l}\left(k_{i}, q_{i}\left(k_{i}\right)\right)$, from the auxiliary function

$$
g_{l}(k, q)=2 i\left[\frac{k}{q}\right]^{l} \frac{q \hat{\jmath}_{l}^{\prime}(q) \hat{h}_{l}^{+}(k)-k \hat{h}_{l}^{+^{\prime}}(k) \hat{\jmath}_{l}(q)}{q\left(\hat{h}_{l}^{+^{\prime}}(k) \hat{h}_{l}^{-}(k)-\hat{h}_{l}^{{ }^{\prime}}(k) \hat{h}_{l}^{+}(k)\right)},
$$

and the $S$-matrix elements from Eq. 20 as

$$
s_{l, i i}^{(0)}(E)=\frac{q_{i} \hat{\jmath}_{l}^{\prime}\left(q_{i}\right) \hat{h}_{l}^{-}\left(k_{i}\right)-k_{i} \hat{h}_{l}^{-^{\prime}}\left(k_{i}\right) \hat{\jmath}_{l}\left(q_{i}\right)}{q_{i} \hat{\jmath}_{l}^{\prime}\left(q_{i}\right) \hat{h}_{l}^{+}\left(k_{i}\right)-k_{i} \hat{h}_{l}^{+^{\prime}}\left(k_{i}\right) \hat{\jmath}_{l}\left(q_{i}\right)} .
$$

Second, in the next order of the DWBA the transition matrix element reads

$$
\begin{aligned}
s_{l, 12}^{(1)}(E)= & \frac{2 v_{12}}{i \delta v \sqrt{k_{1} k_{2}}}\left(\frac{k_{1} k_{2}}{q_{1} q_{2}}\right)^{l+1} \frac{1}{f_{l, 11}^{(0)}\left(k_{1}\right) f_{l, 22}^{(0)}\left(k_{2}\right)} \\
& \times\left[q_{1} \hat{\jmath}_{l-1}\left(q_{1}\right) \hat{\jmath}_{l}\left(q_{2}\right)-q_{2} \hat{\jmath}_{l-1}\left(q_{2}\right) \hat{\jmath}_{l}\left(q_{1}\right)\right] .
\end{aligned}
$$

using Eqs. 25) and (33). Thus, we obtain the interesting result that the transition matrix elements are given in terms of the uncoupled Jost functions. Thus, their zeros determine the poles of the $S$-matrix elements.

\section{B. Exact solution}

Obtaining the full Jost matrix $\mathscr{F}_{l}$ for the coupled square-well analytically is a standard exercise. By matching the wave-function at the intersection, one finds for the diagonal elements

$$
\begin{aligned}
& f_{l, 11}\left(k_{1}\right)=g_{l}\left(k_{1}, q_{+}\right) \cos ^{2} \alpha+g_{l}\left(k_{1}, q_{-}\right) \sin ^{2} \alpha, \\
& f_{l, 22}\left(k_{2}\right)=g_{l}\left(k_{2}, q_{-}\right) \cos ^{2} \alpha+g_{l}\left(k_{2}, q_{+}\right) \sin ^{2} \alpha,
\end{aligned}
$$

and for the off-diagonal elements

$$
\begin{aligned}
& f_{l, 12}\left(k_{1}\right)=\frac{\sin 2 \alpha}{2}\left[\frac{k_{2}}{k_{1}}\right]^{l+1}\left[g_{l}\left(k_{1}, q_{+}\right)-g_{l}\left(k_{1}, q_{-}\right)\right], \\
& f_{l, 21}\left(k_{2}\right)=\frac{\sin 2 \alpha}{2}\left[\frac{k_{1}}{k_{2}}\right]^{l+1}\left[g_{l}\left(k_{2}, q_{+}\right)-g_{l}\left(k_{2}, q_{-}\right)\right],
\end{aligned}
$$

defining a mixing angle $\alpha$ by $\tan 2 \alpha=2 v_{12} / \delta v$. The linearly independent solutions of the Schrödinger equation in the inner region are characterized by the two wavenumbers $q_{ \pm}=\sqrt{k^{2}-\varepsilon_{ \pm}}$, with an energy splitting

$$
\varepsilon_{ \pm}=\frac{1}{2}\left(v_{11}+v_{22} \pm \sqrt{\delta v^{2}+4 v_{12}^{2}}\right) .
$$

Finally, the solution for the $S$-matrix can be found from the Jost matrix by Eq. (17). In particular, we are interested in the off-diagonal element and find

$$
s_{l, 12}(E)=\sqrt{\frac{k_{1}}{k_{2}}} \frac{f_{l, 12}^{*}\left(k_{1}\right) f_{l, 11}\left(k_{1}\right)-f_{l, 11}^{*}\left(k_{1}\right) f_{l, 12}\left(k_{1}\right)}{f_{l, 11}\left(k_{1}\right) f_{l, 22}\left(k_{2}\right)-f_{l, 12}\left(k_{1}\right) f_{l, 21}\left(k_{2}\right)},
$$

with $k_{i} \equiv k_{i}(E)$. Clearly, one recovers the DWBA expression for $s_{l, 12}^{(1)}$ of Eq. 38 from a first order expansion in $\Theta\left(v_{12} / \delta v\right)$.

\section{SINGLE CHANNEL SCATTERING}

The understanding of the coupled channel scattering process is complex as single-channel scattering resonances are intertwined with two channel mixing. It is therefore prudent to dissect the problem and analyze single-channel scattering first. This is achieved by turning off the coupling $v_{12}=0$ between the channels and to study scattering in channel 1 exclusively. Now, we have the freedom to set the threshold energy $\Delta_{11}=0$.

With the goal of parameterizing cross sections and loss rates for cold collisions of atoms analytically, we only consider the lowest $s-, p$-, $d$ - partial-waves. We will not exhaustively study all conceivable cases, but demonstrate the benefits of the pole expansion for certain instances. In particular, for $s$-waves, we consider a potential with a single weakly bound state in Sec. IVA and in Sec. IVB for $p$ - and $d$-waves with a single quasi-bound state. These states dominate the behavior of the cross sections. We will compare exact square-well results to the results from a single-pole expansion due to the weakly bound state for $s$-waves and from a two-pole expansion due to the quasi-bound states for $p$ - and $d$-waves, eventually.

To enhance the visibility of resonances in the examples, we have deliberately chosen different potential depths for each angular momentum channel as listed in Table I] 


\begin{tabular}{lcccc}
\hline \hline Angular momentum & $l$ & $0(\mathrm{~s})$ & $1(\mathrm{p})$ & $2(\mathrm{~d})$ \\
\hline Potential depth & $\kappa_{1}^{2}$ & 4.0 & 9.0 & 19.5 \\
\hline Zero of Jost function & $k_{1}$ & $0.638 i$ & 0.539 & 0.628 \\
& & & $-0.100 i$ & $-0.009 i$ \\
\hline Binding/resonance energy & $E_{r}$ & -0.407 & 0.281 & 0.395 \\
\hline \hline
\end{tabular}

Table I. Potential depth $\kappa_{1}^{2}$, complex zero $k_{1}$ of Jost function $f_{l, 11}^{(0)}\left(k_{1}\right)$ and binding or resonance energy $E_{r}=\operatorname{Re}^{2} k_{1}-\operatorname{Im}^{2} k_{1}$ in the uncoupled channel 1 for different angular momenta. Potential depths were deliberately chosen individually, so that each potential only supports one bound state $(s)$, or one quasibound state $(p, d)$.

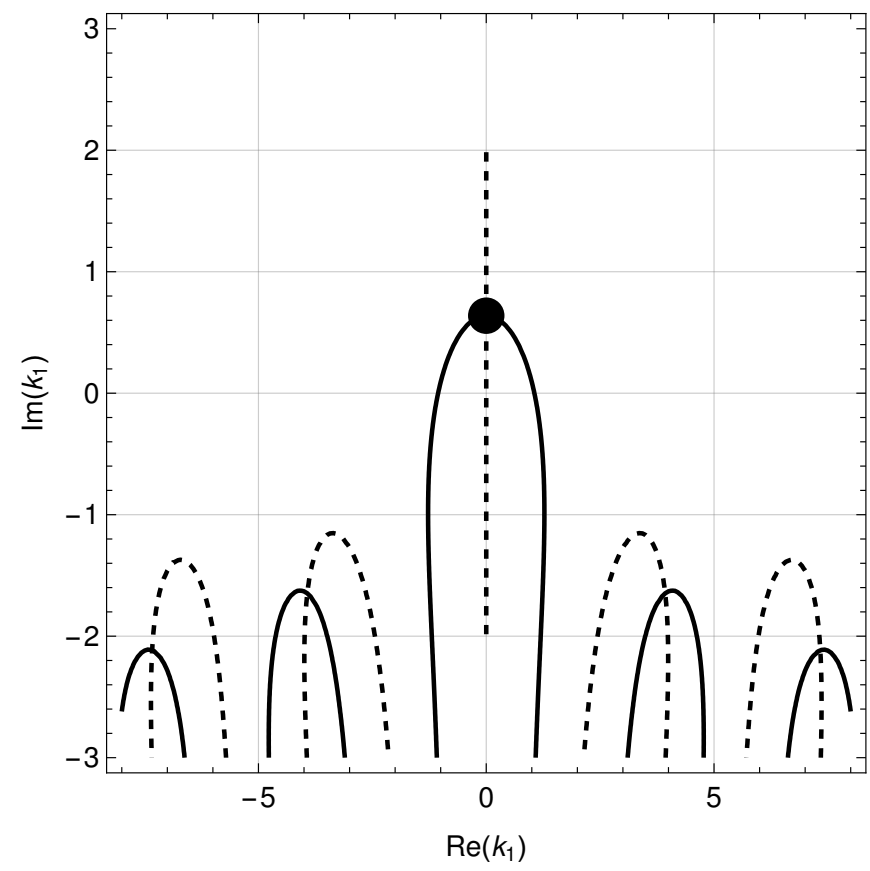

Figure 3. Zero contour lines of the $s$-wave Jost function $\operatorname{Re} f_{0,11}^{(0)}\left(k_{1}\right)=0$ (solid) and $\operatorname{Im} f_{0,11}^{(0)}\left(k_{1}\right)=0$ (dashed) in the complex $k_{1}$-plane for a potential depth $\kappa_{1}^{2}=4$. The black dot indicates the position of a bound state at $k_{1}=i \mathscr{K}_{1}=0.638 i$ and corresponds to a binding energy of $E_{r}=-\mathscr{K}_{1}^{2}=-0.407$.

\section{A. Scattering in the $s$-channel}

Shallow three-dimensional attractive square-wells can have zero, one, or more bound states. By increasing the potential depth continuously, a virtual state with positive energy transforms into a half bound state at zero energy and becomes a bound state with negative energy, eventually. In the following, we will present the exact scattering phase and cross section for $s$-waves and compare these with the pole expansion approximations. a. Exact solution For $l=0$, the Jost function of Eq. (36) reads

$$
f_{0,11}^{(0)}\left(k_{1}\right)=\frac{e^{i k_{1}} \sin q_{1}}{q_{1}}\left(q_{1} \cot q_{1}-i k_{1}\right),
$$

and the $S$-matrix element follows from Eq. (20) as

$$
s_{0,11}^{(0)}\left(k_{1}\right)=e^{-2 i k_{1}} \frac{q_{1} \cot q_{1}+i k_{1}}{q_{1} \cot q_{1}-i k_{1}} .
$$

For real $k_{1}$, it is unimodular $\left|s_{0,11}^{(0)}\right|=1$ and the real scattering phase reads

$$
\eta_{0}\left(k_{1}\right)=n \pi-k_{1}+\arctan \frac{k_{1}}{q_{1} \cot q_{1}},
$$

where Levinson theorem 29] determines the zero energy phase from the number of bound states $n$ in the scattering potential.

b. Single-pole expansion To be specific, we will consider the $s$-potential well-depth in Table 3, which only supports one weakly bound state. It emerges as a zero of the Jost-function $f_{0,11}^{(0)}\left(k_{1}\right)=0$ at $k_{1}=i \mathscr{K}_{1}$ in the complex $k_{1}$-plane. Its zero contours are shown in Fig. 3 . For wave-numbers close to threshold, we assume that the Weierstrass expansion Eq. 28) is dominated by this value. All other zeros contribute to the background scattering phase $\eta_{0}^{p, b}$.

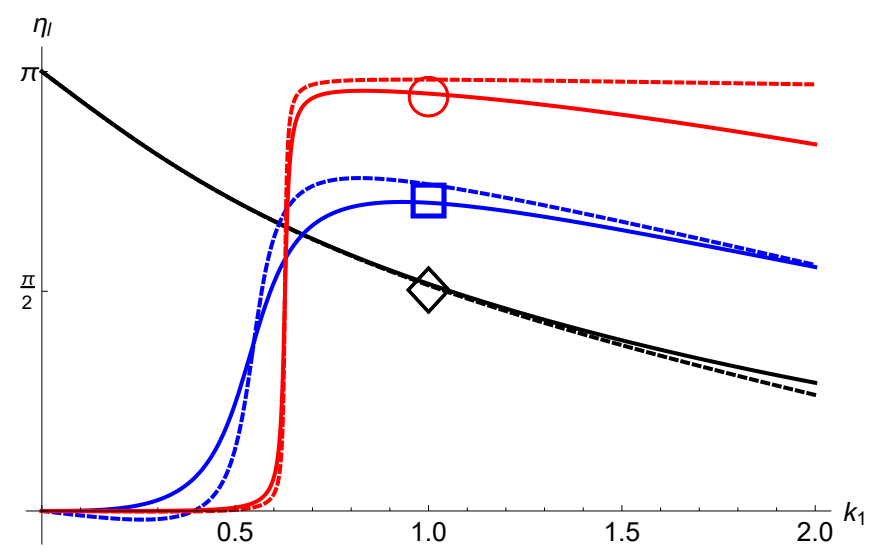

Figure 4. (Color online) Exact scattering phase $\eta_{l}\left(k_{1}\right)$ (solid) and single-pole approximation $\eta_{l}^{p}\left(k_{1}\right)$ (dashed) versus $k_{1}$ for the $l=0$ (black, $\diamond), l=1$ (blue, $\square$ ) and $l=2$ (red, $\circ$ ) partial waves.

Now , the single-pole expansion of the $S$-matrix reads

$$
s_{0,11}^{(0) p}\left(k_{1}\right)=s_{0,11}^{p, b}\left(k_{1}\right) s_{0,11}^{p, r}\left(k_{1}\right)=e^{2 i \eta_{0}^{p, b}} \frac{\mathscr{K}_{1}-i k_{1}}{\mathscr{K}_{1}+i k_{1}},
$$

and the background scattering phase is approximated from a Taylor series at $k_{1}=0$ of Eqs. 46 and 48 up to linear order as

$$
\eta_{0}^{p, b}\left(k_{1}\right)=\frac{1}{2 i} \log \frac{s_{0,11}^{(0)}}{s_{0,11}^{p, r}} \approx n \pi+\left(1-a_{\mathrm{sc}} \mathscr{K}_{1}\right) \frac{k_{1}}{\mathscr{K}_{1}} .
$$


The mathematical phase ambiguity is resolved physically by Levinson's theorem, counting the number of bound states $n \in \mathbb{N}_{0}$ [33. Moreover, we denote the $s$-wave scattering length for the square-well potential [28] as $a_{\mathrm{sc}}=1-\tan \kappa_{1} / \kappa_{1}$. The resonance contribution $\eta_{0}^{p, r}$ can be obtained from the second term in Eq. 48. For real $k_{1}$, it can be transformed into

$$
\eta_{0}^{p, r}\left(k_{1}\right)=-\arctan \frac{k_{1}}{\mathscr{K}_{1}}
$$

using trigonometric relations [34. Consequently, the single-pole expansion of the total scattering phase Eq. 21 is

$$
\eta_{0}^{p}\left(k_{1}\right)=n \pi+\left(1-a_{\mathrm{sc}} \mathscr{K}_{1}\right) \frac{k_{1}}{\mathscr{K}_{1}}-\arctan \frac{k_{1}}{\mathscr{K}_{1}} .
$$

In Fig. 4 the scattering phase $\eta_{0}^{p}$ of the pole expansion is compared to the exact scattering phase $\eta_{0}$. It can be seen that they coincide almost perfectly for small wavenumbers. We have chosen the potential depth parameter to accomodate one bound state, thus the scattering phase approaches $\pi$ at threshold. Analogously, we depict in Fig. 5 the exact $s$-wave elastic cross sections of Eq. (23) with the pole expansion. On the scale shown in the figure, there is hardly any difference.

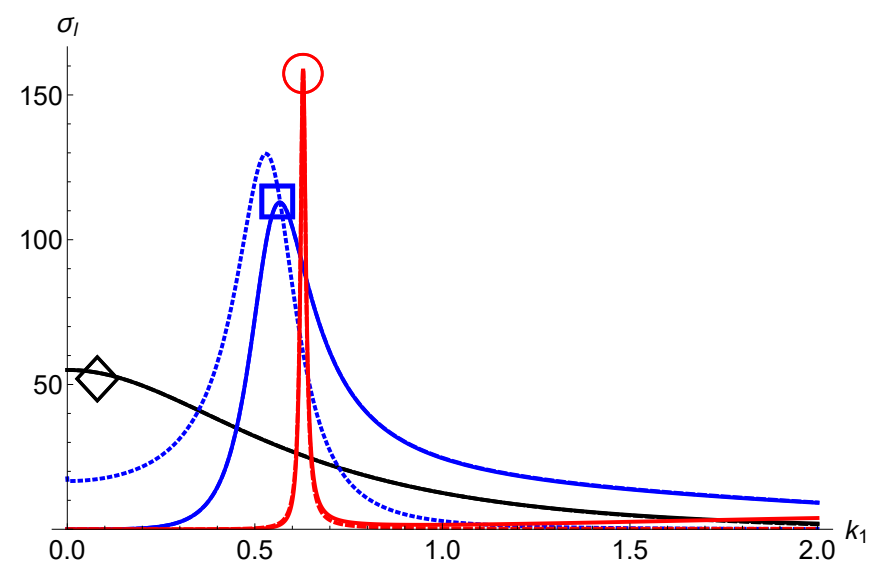

Figure 5. (Color online) The exact partial cross section $\sigma_{l, 11}^{(0)}\left(k_{1}\right)$ (solid) and the single-pole approximation (dashed) versus $k_{1}$ for $l=0$ (black, $\left.\diamond\right), l=1$ (blue, $\square$ ) and $l=2$ $(\mathrm{red}, \circ)$ partial waves are almost indistinguishable. Using only the Breit-Wigner approximation $\sigma_{l=1,11}^{(0) p, r}\left(k_{1}\right)$ (dotted, blue) of Eq. 59p leads to significant deviations in shape and resonance position $k_{r}=\sqrt{E_{r}}=0.530$ (vertical black line).

\section{B. Scattering in the $p$-channel}

In contrast to the $s$-channel, one can not have any virtual states for higher angular momenta. Resonant states with energies below the angular momentum barrier are called quasi-bound states.

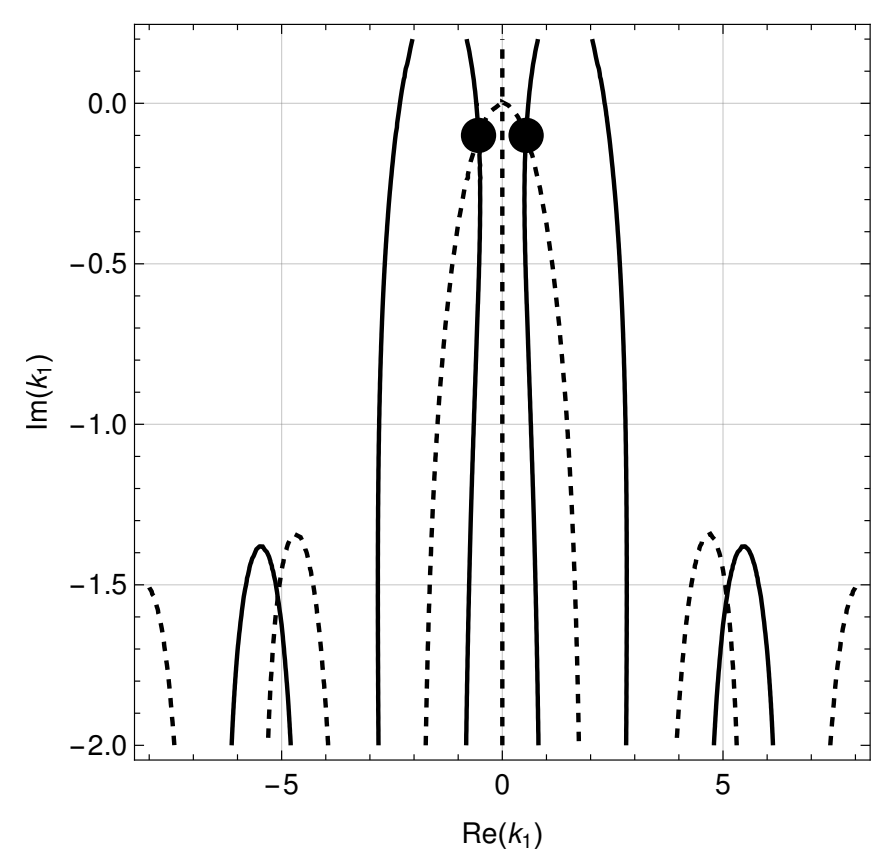

Figure 6. Zero contour lines of the $p$-wave Jost function $\operatorname{Re} f_{1,11}^{(0)}\left(k_{1}\right)=0$ (solid) and $\operatorname{Im} f_{1,11}^{(0)}\left(k_{1}\right)=0$ (dashed) in the complex $k_{1}$-plane for a potential depth $\kappa_{1}^{2}=9$. The black dots indicate the positions $k_{1}=0.539-0.100 i$ and $-k_{1}^{*}$.

a. Exact solution For $l=1$, the Jost function of Eq. 36 becomes

$$
f_{1,11}^{(0)}\left(k_{1}\right)=\frac{e^{i k_{1}} \sin q_{1}}{q_{1}^{3}}\left(k_{1}^{2} q_{1} \cot q_{1}+\kappa_{1}^{2}-i k_{1} q_{1}^{2}\right),
$$

and the uncoupled $S$-matrix element can be derived from Eq. (20) as

$$
s_{1,11}^{(0)}\left(k_{1}\right)=e^{-2 i k_{1}} \frac{k_{1}^{2} q_{1} \cot q_{1}+\kappa_{1}^{2}+i k_{1} q_{1}^{2}}{k_{1}^{2} q_{1} \cot q_{1}+\kappa_{1}^{2}-i k_{1} q_{1}^{2}} .
$$

The corresponding scattering phase is given by

$$
\eta_{1}\left(k_{1}\right)=n \pi-k_{1}+\arctan \frac{k_{1} q_{1}^{2}}{k_{1}^{2} q_{1} \cot q_{1}+\kappa_{1}^{2}} .
$$

Again, the physical scattering phase is determined by the number of bound states $n$ in the $p$-wave potential.

b. Two-pole expansion The $p$-wave potential of Table 3. supports one quasi-bound state. Mathematically, it is represented by two closely spaced zeros $k_{1}=\left\{k_{1},-k_{1}^{*}\right\}$ of the $p$-wave Jost function in the complex $k_{1}$-plane. Its zero contours are shown in Fig. 6. For low values of the wave-number, the Weierstrass expansion of the Jostfunction is dominated by the pair of zeros. All the other zeros contribute cumulatively to the background scattering phase $\eta_{1}^{p, b}$. Then, the two-pole expansion of the $S$ matrix reads

$$
s_{1,11}^{(0) p}\left(k_{1}\right)=s_{1,11}^{p, b} s_{1,11}^{p, r}=e^{2 i \eta_{1}^{p, b}} \frac{\left(k_{1}+k_{1}\right)\left(k_{1}^{*}-k_{1}\right)}{\left(k_{1}^{*}+k_{1}\right)\left(k_{1}-k_{1}\right)},
$$


and $\eta_{1}^{p, b}$ is found from Eqs. 53 and 55

$$
\eta_{1}^{p, b}\left(k_{1}\right)=\frac{1}{2 i} \log \frac{s_{1,11}^{(0)}}{s_{1,11}^{p, r}} \approx n \pi+\frac{2 k_{1} \operatorname{Im} k_{1}}{\left|k_{1}\right|^{2}},
$$

with a Taylor series at threshold $k_{1}=0$ up to linear order. The resonance scattering phase is defined by the second term in Eq. (55) and one finds for real $k_{1}$

$$
\eta_{1}^{p, r}\left(k_{1}\right)=-\arctan \frac{2 k_{1} \operatorname{Im} k_{1}}{\left|k_{1}\right|^{2}-k_{1}^{2}} \text {. }
$$

Then, the total scattering phase of the pole expansion reads

$$
\eta_{1}^{p}\left(k_{1}\right)=n \pi+\frac{2 k_{1} \operatorname{Im} k_{1}}{\left|k_{1}\right|^{2}}-\arctan \frac{2 k_{1} \operatorname{Im} k_{1}}{\left|k_{1}\right|^{2}-k_{1}^{2}} .
$$

In Fig. 4. the scattering phase $\eta_{1}^{p}$ of the pole expansion is compared to the exact solution $\eta_{1}$. The phase vanishes at zero energy as there is no bound state. While there is good qualitative overall agreement, there are noticeable deviations around the resonance position.

The resonant part of the elastic cross section can be evaluated by inserting $s_{1,11}^{p, r}$ in Eq. (23) and one obtains the Breit-Wigner formula 32

$$
\begin{aligned}
\sigma_{1,11}^{(0) p, r}\left(k_{1}\right) & =\frac{12 \pi}{\operatorname{Re}^{2} k_{1}} \frac{\left(\Gamma_{1} / 2\right)^{2}}{\left(k_{1}^{2}-\varkappa_{1}^{2}\right)^{2}+\left(\Gamma_{1} / 2\right)^{2}}, \\
\Gamma_{l} & =4 \operatorname{Re} k_{l} \operatorname{Im} k_{l}, \\
E_{r} & =\varkappa_{l}^{2}=\operatorname{Re}^{2} k_{l}-\operatorname{Im}^{2} k_{l},
\end{aligned}
$$

where we have introduced a resonance energy $E_{r}$ and a width $\Gamma_{l}$. In Fig. 5 , we compare the $p$-wave elastic cross sections Eq. 23 from the exact $S$-matrix $s_{1,11}^{(0)}$ with the pole expansion $s_{1,11}^{(0) p}$ and find excellent agreement. Using only the Breit-Wigner approximation Eq. (59) leads to significant deviations.

\section{Scattering in the $d$-channel}

$D$-wave scattering is qualitatively similar to the $p$-wave results. However, the increasing complexity of the exact solution conceals the physics. Only the pole expansion unveils the essential resonance features.

a. Exact solution For $l=2$, the Jost function of Eq. (36) reads

$$
\begin{aligned}
& f_{2,11}^{(0)}\left(k_{1}\right)=\frac{e^{i k_{1}} \sin q_{1}}{q_{1}^{5}}\left[\left(k_{1}^{4}-3 \kappa_{1}^{2}+3 i k_{1} \kappa_{1}^{2}+k_{1}^{2} \kappa_{1}^{2}\right)\right. \\
& \left.\times q_{1} \cot q_{1}-i\left(k_{1}^{5}+3 i \kappa_{1}^{2}+3 k_{1} \kappa_{1}^{2}+k_{1}^{3} \kappa_{1}^{2}\right)\right]
\end{aligned}
$$

The $S$-matrix element follows from Eq. 20 as

$$
s_{2,11}^{(0)}\left(k_{1}\right)=\frac{f_{2,11}^{(0)}\left(-k_{1}\right)}{f_{2,11}^{(0)}\left(k_{1}\right)},
$$

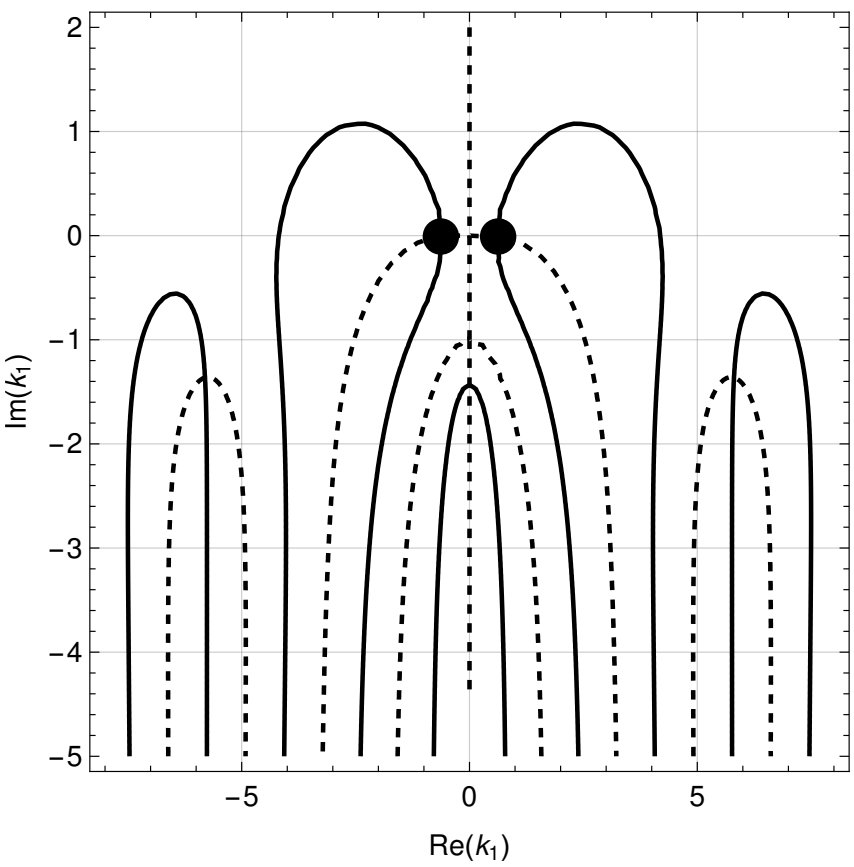

Figure 7. Zero contour lines of the $d$-wave Jost function $\operatorname{Re} f_{2,11}^{(0)}\left(k_{1}\right)=0$ (solid) and $\operatorname{Im} f_{2,11}^{(0)}\left(k_{1}\right)=0$ (dashed) in the complex $k_{1}$ plane for a potential depth $\kappa_{1}^{2}=19.5$. The black dots indicate the positions $k_{2}=0.628-0.009 i$ and $-k_{2}^{*}$.

and for real $k_{1}$ the scattering phase reads

$$
\begin{aligned}
& \eta_{2}\left(k_{1}\right)=n \pi-k_{1} \\
& -\arctan \frac{k_{1}\left[\left(k_{1}^{4}+3 \kappa_{1}^{2}+k_{1}^{2} \kappa_{1}^{2}\right)-3 \kappa_{1}^{2} q_{1} \cot q_{1}\right]}{\left(k_{1}^{4}-3 \kappa_{1}^{2}+k_{1}^{2} \kappa_{1}^{2}\right) q_{1} \cot q_{1}+3 \kappa_{1}^{2}} .
\end{aligned}
$$

b. Two-pole expansion The quasi-bound state of the $d$-wave potential emerges from two zeros $k_{1}=\left\{k_{2},-k_{2}^{*}\right\}$ of the Jost function $f_{2,11}^{(0)}\left(k_{1}\right)$ in the complex $k_{1}$-plane. Its zero contours are shown in Fig. 7. We assume that the pole expansion of the $S$-matrix

$$
\begin{aligned}
s_{2,11}^{(0) p}\left(k_{1}\right) & =s_{2,11}^{p, b}\left(k_{1}\right) \cdot s_{2,11}^{p, r}\left(k_{1}\right) \\
& =e^{2 i \eta_{2}^{p, \mathrm{bg}}\left(k_{1}\right)} \frac{\left(k_{2}+k_{1}\right)\left(k_{2}^{*}-k_{1}\right)}{\left(k_{2}^{*}+k_{1}\right)\left(k_{2}-k_{1}\right)},
\end{aligned}
$$

is dominated by the two poles for low $k_{1}$. The effect of all the other poles contributes to the background scattering phase $\eta_{2}^{p, b}$. Using Eqs. (63) and (65), the background scattering phase is found from a Taylor series at $k_{1}=0$ up to linear order as

$$
\eta_{2}^{p, b}\left(k_{1}\right)=\frac{1}{2 i} \log \frac{s_{2,11}^{(0)}}{s_{2,11}^{p, r}} \approx n \pi+\frac{2 k_{1} \operatorname{Im} k_{2}}{\left|k_{2}\right|^{2}}
$$

It has the same structure as the $p$-wave result $(56)$, which presumably holds also for higher angular momenta. It holds definitively for the resonance phase as the structure of the Weierstrass expansion is identical. Therefore, the 


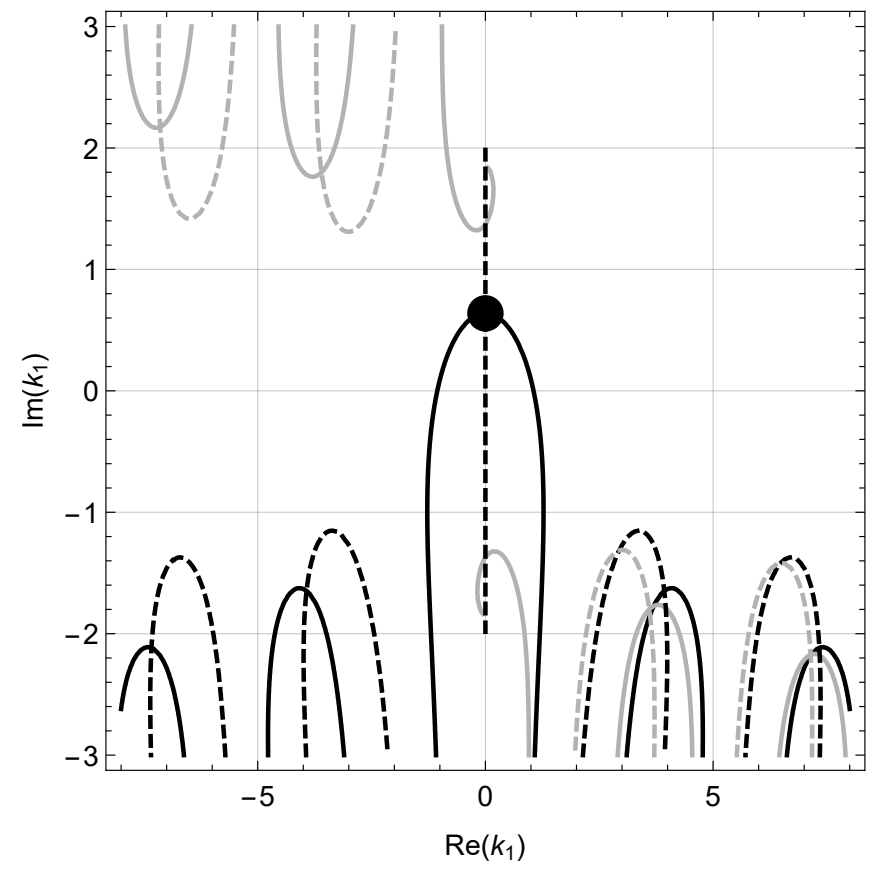

Figure 8. Zero contours lines of the $s$-wave Jost functions in channel 1 and 2: $\operatorname{Re} f_{0,11}^{(0)}\left(k_{1}\right)=0$ (solid, black), $\operatorname{Im} f_{0,11}^{(0)}\left(k_{1}\right)=0$ (dashed, black) and $\operatorname{Re} f_{0,22}^{(0)}\left(k_{1}\right)=0$ (solid, gray), $\operatorname{Im} f_{0,22}^{(0)}\left(k_{1}\right)=0$ (dashed, gray) in the complex $k_{1}$ plane. The black dot indicates the position $k_{1}=i K_{1}=$ $0.638 i$. The potential parameters are given in Table II.

total scattering phase for the pole expansion reads

$$
\eta_{2}^{p}\left(k_{1}\right)=n \pi+\frac{2 k_{1} \operatorname{Im} k_{2}}{\left|k_{2}\right|^{2}}-\arctan \frac{2 k_{1} \operatorname{Im} k_{2}}{\left|k_{2}\right|^{2}-k_{1}^{2}},
$$

analogously to Eq. (58).

In Fig. 4 , the scattering phase $\eta_{2}^{p}$ of the pole expansion is compared to the exact solution $\eta_{2}$. Around the resonance position and for larger $k_{1}, \eta_{2}^{p}$ deviates from the exact solution. In Fig. 5 , the $d$-wave elastic cross sections Eq. 23 found from the exact $S$-matrix $s_{2,11}^{(0)}$ and found from the pole expansion $s_{2,11}^{(0) p}$ are compared. Only for larger $k_{1}$ the solutions start to deviate from each other, the resonant behavior is explained perfectly by the pole expansion.

\section{TWO-BODY LOSS RATES}

Now, we will extent the discussion from the singlechannel case to the coupled two-channel case within the DWBA. We use the same potential parameters for the upper channel as in the single channel case given in Table If. The weakly bound $s$-state in the upper channel as well as the quasi-bound $p$ - and $d$-states will dominate the behavior of the partial two-body loss rates as the channels are only weakly coupled to the loss channels. Given

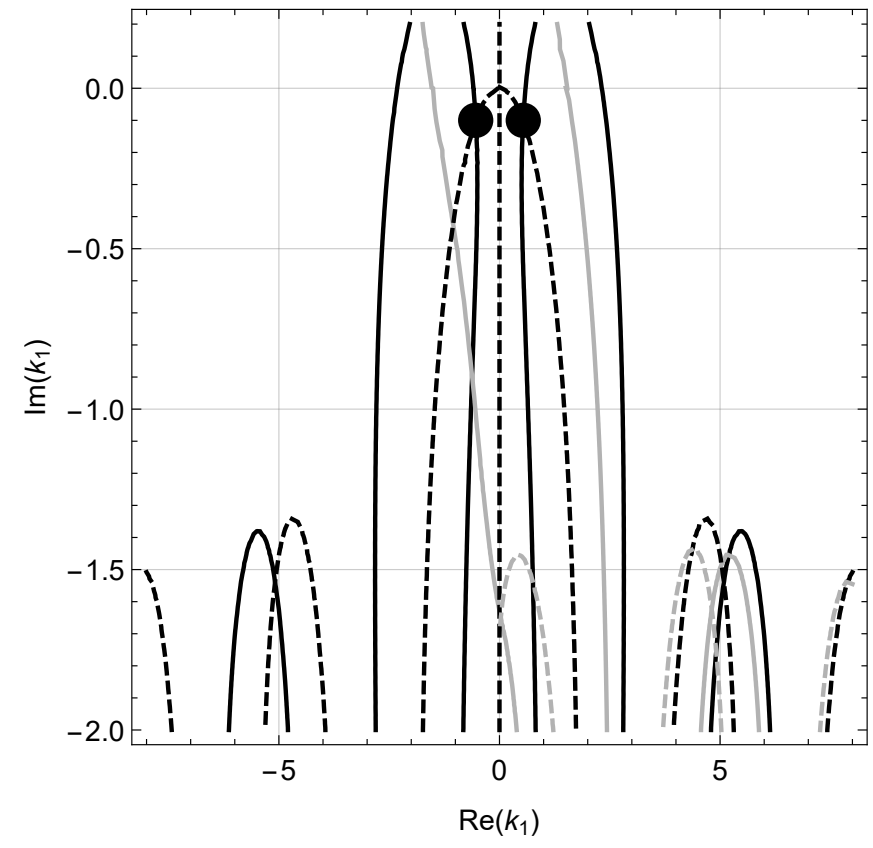

Figure 9. Zero contour lines of $p$-wave Jost function in channels 1 and 2: $\operatorname{Re} f_{1,11}^{(0)}=0$ (solid, black), $\operatorname{Im} f_{1,11}^{(0)}=0$ (dashed, black) and $\operatorname{Re} f_{1,22}^{(0)}=0$ (solid, gray), $\operatorname{Im} f_{1,22}^{(0)}=0$ (dashed, gray) in the complex $k_{1}$ plane. The black dots indicate the positions $k_{1}=0.539-0.100 i$ and $-k_{1}^{*}$. The potential parameters are given in Table II

a sufficiently large separation of channel threshold energies $\Delta_{11} \gg \Delta_{22}$, one can assume that the potential depth $\kappa_{2}^{2}$ of channel 2 does not influence the scattering behavior of channel 1. For simplicity, we have chosen them equal. All other parameters are listed in Table II

\begin{tabular}{lcrrr}
\hline \hline Angular momentum & $l$ & 0 & 1 & 2 \\
\hline Potential depth & $\kappa_{2}^{2}$ & 4.0 & 9.0 & 19.5 \\
Threshold energy channel 1 & $\Delta_{11}$ & 0.0 & 0.0 & 0.0 \\
Threshold energy channel 2 & $\Delta_{22}$ & -3.0 & -3.0 & -3.0 \\
Coupling strength & $v_{12}$ & 0.1 & 0.1 & 0.1 \\
\hline \hline
\end{tabular}

Table II. Potential parameters of two-channel scattering used in the examples.

The transition amplitude between the channels is determined by the $S$-matrix element $s_{l, 12}^{(1)}$ of Eq. 38. Its poles follow from the zeros of the uncoupled Jost functions $f_{l, 11}^{(0)}, f_{l, 22}^{(0)}$ of channel 1 and 2 , respectively.

We present the zero contours of the $s$-wave Jost functions $f_{l, 11}^{(0)}$ and $f_{l, 22}^{(0)}$ in Fig. 8 , and in Fig. 9 the $p$-wave result. It can be seen that the zeros of $f_{l, 22}^{(0)}$ are further away from the origin than the smallest zero(s) of $f_{l, 11}^{(0)}$ marked in blue. A similar picture arises for $l=2$. Therefore, we assume that the pole expansion of $s_{l, 12}^{(1)}$ is 
dominated by the zeros of $f_{l, 11}^{(0)}$ at $k_{1}=i \mathscr{K}_{1}(\mathrm{l}=0)$ and the pair of zeros $k_{1}=\left\{k_{l},-k_{l}^{*}\right\}$ for $l=1,2$. Non-resonant features contribute to the background scattering phase $\eta_{l, 12}^{p, b}$. We obtain for the pole expansion

$$
\begin{aligned}
& s_{0,12}^{(1) p}\left(k_{1}\right)=\frac{a_{0} k_{1}^{\frac{1}{2}} e^{\xi_{0,12}^{p, b}\left(k_{1}\right)}}{\mathscr{K}_{1}+i k_{1}}, \\
& s_{l, 12}^{(1) p}\left(k_{1}\right)=\frac{a_{l} k_{1}^{\frac{2 l+1}{2}} e^{\xi_{l, 12}^{p, b}\left(k_{1}\right)}}{\left(k_{l}^{*}+k_{1}\right)\left(k_{l}-k_{1}\right)},
\end{aligned}
$$

where the second line holds for $l>0$ and the background attenuation coefficients are given by

$$
\begin{aligned}
\xi_{0,12}^{p, b}\left(k_{1}\right)= & \log \left[s_{0,12}^{(1)}\left(k_{1}\right) \frac{\left(\mathscr{K}_{1}+i k_{1}\right)}{a_{0} \sqrt{k_{1}}}\right] \\
& =\frac{1}{2}\left(b_{0} k_{1}+c_{0} k_{1}^{2}+\ldots\right), \\
\xi_{l, 12}^{p, b}\left(k_{1}\right)= & \log \left[s_{l, 12}^{(1)}\left(k_{1}\right) \frac{\left(k_{l}^{*}+k_{1}\right)\left(k_{l}-k_{1}\right)}{a_{l} k_{1}^{\frac{2 l+1}{2}}}\right] \\
= & \frac{1}{2}\left(b_{l} k_{1}+c_{l} k_{1}^{2}+\ldots\right) .
\end{aligned}
$$

Here, a Taylor expansion of the background scattering phases around $k_{1}=0$ yields expansion coefficients $a_{l}, b_{l}, c_{l}, \ldots$, which depend on the potential parameters and the pole positions.

From this expansion, one can obtain the two-body loss rates of Eq. 26). In lowest order, they read

$$
\begin{aligned}
& \beta_{0}^{(1) p}\left(k_{1}\right)=\frac{2 \pi\left|a_{0}\right|^{2}}{k_{1}^{2}+\mathscr{K}_{1}^{2}}, \\
& \beta_{l}^{(1) p}\left(k_{1}\right)=\frac{2 \pi(2 l+1)\left|a_{l}\right|^{2}}{\left(k_{1}^{2}-\varkappa_{l}^{2}\right)^{2}+\left(\Gamma_{l} / 2\right)^{2}} k_{1}^{2 l},
\end{aligned}
$$

An important feature of the pole expansion of the twobody loss rates in the Eqs. $(72)$ is the power law behavior $k_{1}^{2 l}$. This is known as the Wigner threshold behavior [3537.

In Fig. 10, we compare the partial two-body loss rates of the DWBA Eq. 26) with the pole expansion Eqs. 72. The pole expansion describes the low-energy and the resonant behavior of the two-body loss rates very well and starts to deviate from the DWBA solution only beyond the resonance positions.

\section{ANALYSIS OF SYNTHETIC SCATTERING DATA WITH SHAPE FUNCTIONS}

Here, we employ the pole expansion of the partial twobody loss rates Eq. 72 to analyze the synthetic scattering data of [27] for heteronuclear ${ }^{20} \mathrm{Ne}^{21} \mathrm{Ne} \mathrm{PI}$ and $\mathrm{AI}$ collisions. In the simulations of [27, the two-body loss rate $\beta$ was calculated in terms of a partial-wave decomposition

$$
\beta=\frac{1}{2} \sum_{l} \beta_{l}
$$

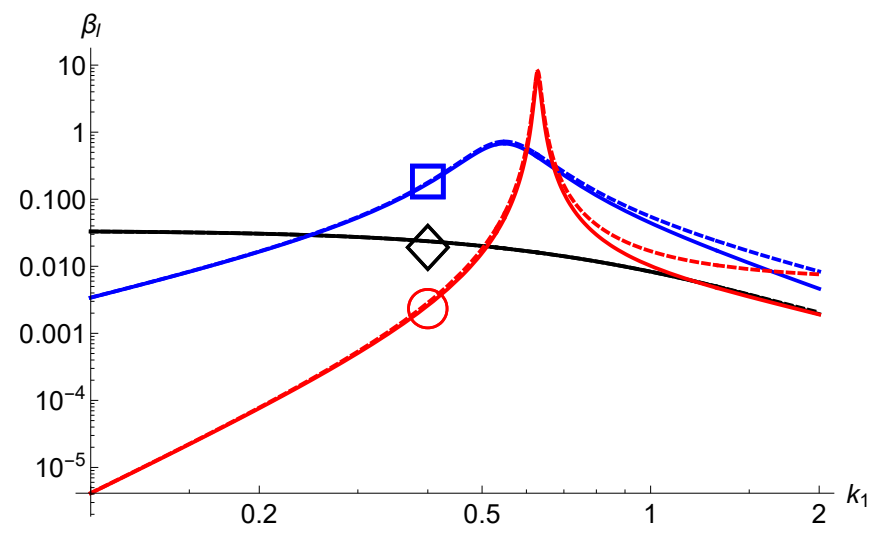

Figure 10. (Color online) DWBA partial-wave two-body loss rates $\beta_{l}^{(1)}$ (solid) and the single-pole approximation $\beta_{l}^{(1) p}$ (dashed) versus channel wave-number $k_{1}$ for $l=0$ (black, $\diamond), l=1$ (blue, $\square), l=2$ (red, ○) for the potential parameters of Table II.

with the partial two-body loss rates $\beta_{l}$. The factor $1 / 2$ accounts for the collisions of non-identical particles [38]. In the experimental temperature regime $T \simeq 1 \mathrm{mK}$, only few partial-waves $l \leq 2$ contribute to the total loss rate.

In order to obtain a high quality interpolation of the two-body loss rates of the available synthetic scattering data, we have to consider the higher order corrections of Eqs. (70/71) in the two-body loss rates of Eq. 26) and find

$$
\begin{aligned}
& \beta_{0}^{p}(k)=\frac{2 \pi\left|a_{0}\right|^{2}}{k^{2}+\mathscr{K}_{1}^{2}} e^{\operatorname{Re} c_{0} k^{2}}, \\
& \beta_{1}^{p}(k)=\frac{6 \pi\left|a_{1}\right|^{2}}{\left(k^{2}-\varkappa_{1}^{2}\right)^{2}+\left(\Gamma_{1} / 2\right)^{2}} k^{2} e^{\operatorname{Re} c_{1} k^{2}}, \\
& \beta_{2}^{p}(k)=\frac{10 \pi\left|a_{2}\right|^{2}}{\left(k^{2}-\varkappa_{2}^{2}\right)^{2}+\left(\Gamma_{2} / 2\right)^{2}} k^{4} e^{\operatorname{Re}\left(c_{2} k^{2}+e_{2} k^{4}\right)} .
\end{aligned}
$$

In the additional attenuation factors of the two-body loss rates all odd power of $k_{1}$ vanish as the coefficients $b_{l}, d_{l}, \ldots$ are purely imaginary.

A least square fit of the partial two-body loss rates in the Eqs. (74a), (74b), (74c to the synthetic data of Ref. [27] leads to the coefficients given in Table III.

In Fig. 11. we compare the synthetic data with the optimal fits from the shape functions obtained from the pole expansion. We also included the single available experimental data point from Ref. [11 to the picture. It can be seen that the pole expansion agrees very well with the synthetic data as well as with the experimental data point. Technically speaking, the two-body loss rates should be averaged thermally to match the experimental data point. However, the width of the relative velocity distribution in the thermal gas is large compared to the widths of the two-body loss rates. Moreover, the error bar for the experimental data point is large, too. Therefore, we neglect the effects of thermal averaging. 


\begin{tabular}{crrr}
\hline \hline & $l=0$ & $l=1$ & $l=2$ \\
\hline$a_{l}$ & 0.1477 & 0.1326 & 2.3141 \\
$\operatorname{Re} c_{l}$ & 57.3641 & 76.8736 & -64.7843 \\
$\operatorname{Re} e_{l}$ & - & - & -642.3825 \\
$\mathscr{K}_{l}$ & 0.0408 & - & - \\
$\operatorname{Re} k_{l}$ & - & $1.5 e^{-10}$ & 0.0304 \\
$\operatorname{Im} k_{l}$ & - & -0.0198 & -0.0100 \\
\hline \hline
\end{tabular}

Table III. Expansion coefficients and pole positions found from fit of $\beta_{l}^{p}$ to the synthetic data.

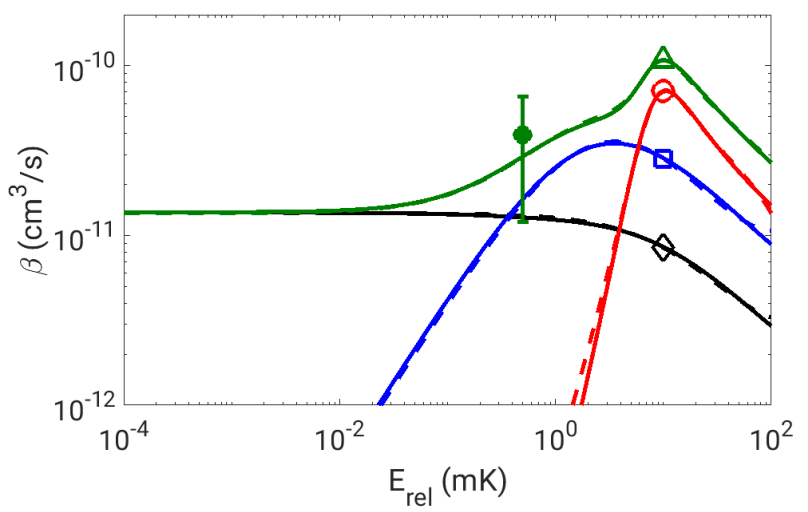

Figure 11. (Color online) Synthetic scattering two-body loss rates $\beta^{\text {synt }}$ (solid, green, $\triangle$ ) and pole expansion $\beta^{p}$ (dashed, green, $\triangle$ ) with the coefficients of Table III versus relative collisions energy $E_{\mathrm{rel}}=\hbar k^{2} / 2 \mu$ in units of $k_{B}$ for ${ }^{21} \mathrm{Ne}-{ }^{20} \mathrm{Ne}$ collisions. Additionally shown are the partial-wave contributions $1 / 2 \times \beta_{l}^{\text {synt }}$ [solid, (black, $\left.\diamond\right),($ blue, $\square),($ red, $\left.\circ)\right], 1 / 2 \times \beta_{l}^{p}$ [dashed, (black, $\diamond)$, (blue, $\square)$, (red, ○)] for $l=0,1,2$, respectively, and experimental data point (green filled circle with error bars).

\section{CONCLUSION}

In this article, we have presented a coupled twochannel model for the reactive collisions of atoms at low collision energies. Transitions from the elastic scattering channel to the lower ionization channel models loss of atoms in two-body collisions, as observed in autoionization and Penning ionization processes. In particular, we study the two-channel square-well model. On one hand, this model can be solved in closed form and, on the other hand, one can use the pole approximation to obtain physically motivated shape functions from it. To extract useful approximations, we employed the distorted-wave Born approximation and studied the poles in the complex $k_{1}$ plane for the lowest $s$-, $p$ - and $d$-partial-waves. From this analysis, we obtain simple analytic expressions for the partial two-body loss rates. Fitting these analytic two-body loss rates to available synthetic scattering data [27] on cold heteronuclear ${ }^{20} \mathrm{Ne}^{-21} \mathrm{Ne}$ collisions gives very good agreement and also matches the experimental data point of Ref. [11.

\section{ACKNOWLEDGEMENTS}

We are very grateful to E. Tiesinga, S. Kotochigova, P. S. Julienne, and C. Williams for their hospitality at NIST and for inspiring discussions. In particular, we thank S. Kotochigova for providing the Ne* molecular potentials to us. Moreover, we thank A. Martin, J. Schütz and G. Birkl for numerous helpful discussions and providing experimental data. RW acknowledge gratefully travel support from the German Aeronautics and Space Administration (DLR) through grant 50 WM 1137.

\section{Appendix A: Riccati-Bessel functions}

Bessel function are a core element of three-dimensional scattering theory. In order to avoid definitional ambiguities, we use the Riccati-Hankel functions

$$
\hat{h}_{l}^{ \pm}(z) \equiv \hat{n}_{l}(z) \pm i \hat{\jmath}_{l}(z)
$$

as in Ref [29], where $\hat{\jmath}_{l}(z)$ is the Riccati-Bessel function and $\hat{n}_{l}(z)$ is the Riccati-Neumann function 39]

$$
\begin{aligned}
& \hat{\jmath}_{l}(z) \equiv \sqrt{\frac{\pi z}{2}} J_{l+\frac{1}{2}}(z), \\
& \hat{n}_{l}(z) \equiv(-1)^{l} \sqrt{\frac{\pi z}{2}} J_{-l-\frac{1}{2}}(z),
\end{aligned}
$$

with the Bessel function of the first kind $J_{l}(z)$. The Riccati-Hankel functions have the symmetry properties

$$
\hat{h}_{l}^{ \pm}(-z)=(-1)^{l+1} \hat{h}_{l}^{\mp}(z) .
$$

The behavior of $\hat{\jmath}_{l}(z)$ for small arguments $z$ is given by

$$
\hat{\jmath}_{l}(z) \underset{z \rightarrow 0}{\longrightarrow} \frac{z^{l+1}}{(2 l+1) ! !} .
$$

\section{Appendix B: Analytic pole approximation}

One can determine the zeros of the Jost function $f_{l, 11}^{(0)}$ in the complex $k_{1}$ plane either numerically or analytically by introducing simple approximations. In the case of the $s$-wave Jost function Eq. 45, one can use complex transformations $k_{1} \equiv z \kappa_{1}, z \equiv-i \cos w$, to find

$$
\kappa_{1} \sin w=w+n \pi, \quad n \in \mathbb{N}_{0}^{+} .
$$

By solving this equation for $w$ and for all $n$, we obtain all zeros of $f_{0,11}^{(0)}$. For the zeros close to the origin of the complex plane, we can assume $\left|k_{1}\right| / \kappa_{1} \ll 1$ and it follows 
$w \simeq \pi / 2$. A Taylor series of (B1) at $w=\pi / 2$ to second order leads to

$$
w_{ \pm}=\frac{-1+\pi \kappa_{1} \pm \sqrt{1-\pi \kappa_{1}-2 n \pi \kappa_{1}+2 \kappa_{1}^{2}}}{\kappa_{1}} .
$$

The zero of the Jost function due to the first bound state of the potential is given by $w_{+}$and $n=0$, the zero of the Jost function due to the second bound state by $w_{+}$ and $n=1$ and so forth. For $N$ bound states present in the scattering potential, the zero of the Jost function of the first virtual state is given by $w_{+}$for $n=N$, for the second virtual state by $w_{+}$for $n=N+1$ and so forth up to $n=N+N^{\prime}-1$ for $N^{\prime}$ virtual states present. The zeros of the Jost function due to scattering resonances are given by the two solutions $w_{ \pm}$for $n>N+N^{\prime}-1$.

For $\kappa_{1}=4$ and $n=0$, we find $w_{+}=1.895$ from Eq. (B2). Resubstitution leads to a zero of the Jost function at $k_{1}=i \mathscr{K}_{1}$ with $\mathscr{K}_{1}=0.637$. The numerically determined value is given by $\mathscr{K}_{1}=0.638$.
[1] F. Penning, Naturwissenschaften 15, 818 (1927).

[2] P. E. Siska, Rev. Mod. Phys. 65, 337 (1993)

[3] W. Vassen, C. Cohen-Tannoudji, M. Leduc, D. Boiron, C. I. Westbrook, A. Truscott, K. Baldwin, G. Birkl, P. Cancio, and M. Trippenbach, Rev. Mod. Phys. 84, 175 (2012)

[4] H. Friedrich, Scattering Theory (Springer Verlag Berlin Heidelberg, 2013).

[5] R. J. W. Stas, J. M. McNamara, W. Hogervorst, and W. Vassen, Phys. Rev. A 73, 032713 (2006).

[6] J. M. McNamara, R. J. W. Stas, W. Hogervorst, and W. Vassen, Phys. Rev. A 75, 062715 (2007)

[7] A. S. Dickinson, J. Phys. B 40, F237 (2007)

[8] C. Orzel, M. Walhout, U. Sterr, P. S. Julienne, and S. L. Rolston, Phys. Rev. A 59, 1926 (1999)

[9] H. Katori, H. Kunugita, and T. Ido, Phys. Rev. A 52, R4324 (1995).

[10] P. Spoden, M. Zinner, N. Herschbach, W. J. van Drunen, W. Ertmer, and G. Birkl, Phys. Rev. Lett. 94, 223201 (2005)

[11] J. Schütz, T. Feldker, H. John, and G. Birkl, Phys. Rev. A 86, 022713 (2012)

[12] J. Schütz, A. Martin, C. Cop, R. Walser, and G. Birkl, to be published (2017).

[13] M. Berglund and M. Wieser, Pure and Applied Chemistry 83, 397 (2009)

[14] S. Kotochigova, E. Tiesinga, and I. Tupitsyn, Phys. Rev. A 61, 042712 (2000).

[15] S. Kotochigova, private comm. (2015).

[16] A. Derevianko and A. Dalgarno, Phys. Rev. A 62, 062501 (2000).

[17] Z. Idziaszek and P. S. Julienne, Phys. Rev. Lett. 104, $113202(2010)$

[18] K. Jachymski, M. Krych, P. S. Julienne, and Z. Idziaszek, Phys. Rev. Lett. 110, 213202 (2013)

[19] K. Jachymski, M. Krych, P. S. Julienne, and Z. Idziaszek, Phys. Rev. A 90, 042705 (2014).

[20] Z. Idziaszek, A. Simoni, T. Calarco, and P. S. Julienne, New J. Phys. 13, 083005 (2011).

[21] P. S. Julienne, Faraday Discuss. 142, 361 (2009).
[22] G. Quemener and J. L. Bohn, Phys. Rev. A 81, 022702 (2010)

[23] Z. Idziaszek, G. Quéméner, J. L. Bohn, and P. S. Julienne, Phys. Rev. A 82, 020703 (2010)

[24] S. Kotochigova, New J. Phys. 12, 073041 (2010).

[25] P. S. Julienne, T. M. Hanna, and Z. Idziaszek, Phys. Chem. Chem. Phys. 13, 19114 (2011)

[26] M. D. Frye, P. S. Julienne, and J. M. Hutson, New Journal of Physics 17, 045019 (2015)

[27] C. Cop, A. Martin, G. Birkl, and R. Walser, to be published (2017).

[28] J. Walraven, Elements of Quantum Gases: Thermodynamic and Collisional Properties of Trapped Atomic Gases (Lecture Notes, 2009).

[29] J. Taylor, Scattering Theory: The Quantum Theory of Nonrelativistic Collisions (New York: Dover Publications, 2006).

[30] E. Freitag and R. Busam, Complex analysis, 2nd ed., Universitext (Springer, Berlin [u.a.], 2009).

[31] J. B. Conway, Functions of one complex variable : 1., 2nd ed., Graduate texts in mathematics, Vol. 11 (Springer, New York [u.a.], 2010).

[32] R. Newton, Scattering Theory of Waves and Particles (2nd edition) (New York: Dover Publications, Inc., 2002).

[33] For simplicity, we want to consider an integer number of bound states and do not want to discuss the well known limit of half-bound states.

[34] For a real angle $\alpha$, following useful identity has been employed repeatedly: $\arctan \alpha=\frac{1}{2 i} \log \frac{1+i \alpha}{1-i \alpha}+n \pi$.

[35] P. S. Julienne and F. H. Mies, J. Opt. Soc. Am. B 6, 2257 (1989).

[36] H. A. Bethe, Phys. Rev. 47, 747 (1935).

[37] E. P. Wigner, Phys. Rev. 73, 1002 (1948)

[38] J. Weiner, V. S. Bagnato, S. Zilio, and P. S. Julienne, Reviews of Modern Physics 71, 1 (1999)

[39] M. Abramowitz and I. Stegun, Handbook of mathematical functions (Dover Publications, Inc., 1965). 